\title{
Effectiveness of quadrivalent human papillomavirus vaccine for the prevention of cervical abnormalities: case-control study nested within a population based screening programme in Australia
}

\begin{abstract}
Objective To measure the effectiveness of the quadrivalent human papillomavirus (HPV) vaccine against cervical abnormalities four years after implementation of a nationally funded vaccination programme in Queensland, Australia.

Design Case-control analysis of linked administrative health datasets. Setting Queensland, Australia.

Participants Women eligible for free vaccination (aged 12-26 years in 2007) and attending for their first cervical smear test between April 2007 and March 2011. High grade cases were women with histologically confirmed high grade cervical abnormalities $(n=1062)$ and "other cases" were women with any other abnormality at cytology or histology $(n=10$ 887). Controls were women with normal cytology ( $n=96404)$.

Main outcome measures Exposure odds ratio (ratio of odds of antecedent vaccination (one, two, or three vaccine doses compared with no doses) among cases compared with controls), vaccine effectiveness ((1-adjusted odds ratio $) \times 100)$, and number needed to vaccinate to prevent one cervical abnormality at first screening round. We stratified by four age groups adjusted for follow-up time, year of birth, and measures of socioeconomic status and remoteness. The primary analysis concerned women whose first ever smear test defined their status as a case or a control.
\end{abstract}

Results The adjusted odds ratio for exposure to three doses of HPV vaccine compared with no vaccine was 0.54 (95\% confidence interval 0.43 to 0.67$)$ for high grade cases and 0.66 ( 0.62 to 0.70$)$ for other cases compared with controls with normal cytology, equating to vaccine effectiveness of $46 \%$ and $34 \%$, respectively. The adjusted numbers needed to vaccinate were 125 (95\% confidence interval 97 to 174 ) and 22 (19 to 25), respectively. The adjusted exposure odds ratios for two vaccine doses were 0.79 (95\% confidence interval 0.64 to 0.98 ) for high grade cases and 0.79 ( 0.74 to 0.85 ) for other cases, equating to vaccine effectiveness of $21 \%$.

Conclusion The quadrivalent HPV vaccine conferred statistically significant protection against cervical abnormalities in young women who had not started screening before the implementation of the vaccination programme in Queensland, Australia.

\section{Introduction}

Two prophylactic human papillomavirus (HPV) vaccines are currently available worldwide. Phase III studies have shown that both the quadrivalent vaccine, targeted against HPV types $6,11,16$, and 18 , and the bivalent vaccine, targeted against types 16 and 18, prevent cervical lesions associated with the respective HPV types. ${ }^{1-4}$ Some cross protection against other HPV types has also been shown. ${ }^{5-7}$ The quadrivalent vaccine 
also prevents high grade vulval and vaginal lesions and genital warts in women, as well as genital warts and high grade anal disease in men. ${ }^{12}$ Efficacy against cervical abnormalities was greatest in the population of women who tested negative for the relevant vaccine HPV types at enrolment, as the vaccine does not seem to impact on the clinical course of existing infections. Although clinical trials conducted in controlled settings have shown the efficacy of the quadrivalent vaccine, less is known about the vaccine's effectiveness when delivered to the broader population.

Prophylactic HPV vaccination programmes have been implemented in over 40 countries. $^{8}$ Australia was the first country to implement a publicly funded national vaccination programme with the quadrivalent HPV vaccine in April 2007. As well as initiating an ongoing programme for 12 and 13 year old girls, an extensive catch-up programme was implemented and ran until December 2009. The school based catch-up programme targeted 12-17 year olds, whereas the community catch-up phase offered vaccination to women aged 18 to 26 years in general practice and community settings. The school based programme achieved vaccination rates of $84 \%, 79 \%$, and $70 \%$ for one, two, and three doses, respectively, while the corresponding rates for the community programme were $64 \%$, $53 \%$, and $33 \%$. ${ }^{9}$ We estimated the effectiveness of full and partial courses of quadrivalent HPV vaccine against high grade and other cervical abnormalities in the population of Queensland women targeted by both the school and the community based catch-up vaccination programmes in the first four years after their introduction.

\section{Methods}

\section{Study overview and population}

We performed a case-control analysis using linked, anonymised data obtained from population registers in Queensland, Australia, for a four year period after the introduction of the HPV vaccination programme in April 2007. The study population comprised all female Queensland residents who attended for their first ever cervical smear test between 1 April 2007 and 31 March 2011 and who had been eligible for HPV vaccination during the nationally funded catch-up programme - that is, those born between July 1980 and July 1997.

Our primary objective was to estimate the effectiveness of the quadrivalent vaccine in the population of sexually naïve young women with no previous infection. Information on sexual history was not available from study participants. The Australian national cervical screening programme recommends that cervical screening should start between the ages of 18 and 20 in women who have ever been sexually active, or one or two years after first having sexual intercourse, whichever is later. ${ }^{10}$ To best realise our objective using the data available, we restricted our study to women who presented for their first smear test.

\section{Sampling frame}

We determined case-control status from cytology and histology test results as recorded on the Queensland Health Pap Smear Register. This depository is an "opt off" register, which has stored results since $1999^{11}$ and is estimated to contain data for 98.5-99.5\% of all Queensland women participating in the cervical screening programme. ${ }^{12}$ Cervical cytology results are coded by reporting laboratories according to the Australian standard modified Bethesda coding schedule and electronically forwarded to the register.

\section{Case-control definitions}

We defined two case groups (table $1 \Downarrow$ ) based on the first abnormal test result returned by a woman during the study period. High grade cases were those women who had a high grade cervical abnormality (cervical intraepithelial neoplasia 2 or adenocarcinoma in situ, or worse) confirmed by histology during the study period. We took the index date to be the date of the abnormal cytology test result immediately preceding the histology test result satisfying the high grade case definition, because from that point women entered a phase of diagnostic testing. "Other cases" were those women who did not meet the high grade case definition but had any other abnormality (either a low grade abnormality at histology or an abnormal cytology result that was not confirmed by histology). Thus, other cases included women with high grade cytological abnormalities who did not have subsequent histological testing during the study period, as well as women whose subsequent histology identified only a low grade abnormality or a negative finding. We classified cases with simultaneous squamous and endocervical abnormalities according to the endocervical abnormality. Endocervical abnormalities were judged to be more important because they are rare, always an indication for diagnostic testing, and of particular interest because cervical screening is not sensitive in detecting and preventing adenocarcinoma. The index date for other cases was the date of the first cytological abnormality to occur in the study period.

We assigned control status to the remaining women. By definition all controls had only negative cytology results during the study period. A negative cytology test result consisted of a negative result for the squamous component and negative or unsatisfactory result for the endocervical component. A woman provided control data only once.

\section{Source of exposure information and exposure definitions}

Our exposure of interest was receipt of the HPV vaccine before the index date, as recorded on the Queensland Health Vaccination Information Vaccination Administration System. The register includes several mandatory fields: name, date of birth, address, practitioner and clinic details, date of vaccination, type of vaccine, and dose number. Dose dates are recorded against the relevant dose number, as reported by the healthcare provider. A complete vaccination course consists of three doses over a 4-6 month period. We considered a vaccine dose to be valid if administered in line with national guidelines for minimum intervals, and a woman to be fully vaccinated if no more doses were clinically indicated. ${ }^{13}$ We also excluded individual doses that were subject to a cold chain breach (that is, the vaccine stored outside of the recommended temperature range) or were duplicate vaccine doses (where the same dose date was recorded more than once per woman). We defined women as unvaccinated (no doses), partially vaccinated (one or two doses), or fully vaccinated ( $\geq 3$ doses) where receipt of vaccine doses occurred at any point before the index date.

\section{Data linkage}

Extracts from the Queensland cervical smear register and Queensland vaccination administration system were sent for linkage to Queensland Health Data Linkage Unit. The unit used the LinkageWiz software package to probabilistically identify potentially matching records for each woman. Weighting scores were assigned to matching variables, including full name, sex, date of birth, and address. The linkage generated a total of 287 224 potential matches (pairs) with weighting scores between 
12 and 35. Of these, 249885 pairs $(87.0 \%)$ were automatically accepted because of a weighting score of more than 19 or an exact match on full name and address or full name and date of birth. The remaining 37339 pairs were reviewed; 27257 $(73.0 \%, 9.5 \%$ overall) were accepted as true pairs and 10082 $(27.0 \%, 3.5 \%$ overall) were rejected. We received an anonymised linked dataset.

\section{Ineligibility and exclusions}

We excluded women whose first record in the cervical smear register was for a histology test result, and women who had any of the following characteristics: a recorded histology test during the study period that was not preceded by a record of an abnormal cytology test, duplicate records in the vaccination administration system that could not be combined into one record, inconsistent vaccination records (for example, an unreliable sequence of vaccination dose dates), or a postcode that was not possible to assign to a socioeconomic or remoteness category. We excluded cytology and histology tests that were reported as unsatisfactory for clinical reporting purposes. The figure $\Downarrow$ and supplementary figure 1 on bmj.com show the numbers of women in each of these exclusion categories.

\section{Covariates}

For measures of remoteness and socioeconomic status, we assigned women to a category according to the 2006 Australian Bureau of Statistics (ABS) Remoteness Area (a measure of the remoteness of a location from the services provided by large towns or cities) and to a 2006 ABS Index of Relative Socioeconomic Disadvantage quintile based on current residential postcode on the Queensland cervical smear register. We calculated the median follow-up time from study start date to index date for the study population overall and assigned women to follow-up time quartiles. We included year of birth as a covariate. No information was available on other potential confounders, such as lifestyle factors, from the administrative datasets.

\section{Statistical analysis}

Analyses were conducted for all women and within strata of four age groups (at 2007): 11-14 years, 15-18 years, 19-22 years, and 23-27 years. We restricted our primary analysis to those women who had no cytology tests before their index date (women whose first ever smear test result defined their status as a case or control). This was because the number of previous cytology tests differed between cases and controls. Our secondary analysis comprised cases and controls with one or more cytology tests before their index date. For the secondary analysis, we randomly selected an index date for controls from all negative test results to minimise any opportunity for exposure bias. Such a bias might otherwise have occurred had we used the last test result available, allowing control women to be in the study longer and therefore have more opportunity to become vaccinated than cases.

Our measure of association was the exposure odds ratio- that is, the ratio of exposure odds among cases to the exposure odds among controls. We estimated the exposure odds ratio for each vaccination dose compared with no vaccination using multinomial logistic regression models for each of the two mutually exclusive case categories (high grade cases, other cases) compared with controls. We adjusted for potential confounding by socioeconomic status, remoteness, age, and follow-up time. We estimated vaccine effectiveness and associated $95 \%$ confidence intervals using the formula (1-adjusted odds ratio) $\times 100$. We calculated approximate point and interval estimates of the number needed to be vaccinated to prevent one cervical abnormality at first screening round using multiple logistic regression with adjustment for confounding variables, according to the method of Bender and Blettner. ${ }^{14}$ We used SAS statistical software (version 9.4) for all data cleaning and analyses.

\section{Sensitivity analyses}

We tested the impact of excluding cases and controls in whom the time interval (latent period) between date of last vaccination and index date was shorter than nominated latent periods. We assumed putative latent periods of 30,180, and 365 days based on uncertainties about the timing of immune responses to HPV vaccination and the interval between infection and neoplasia. ${ }^{15} 16$ Because the date of origin of a cervical lesion cannot be known with certainty, it is possible that our decision to define the event date as the cytology test result immediately preceding the abnormal histology result may have introduced bias. We therefore repeated our secondary analyses using the date of the first abnormal cytology test result as the index date for high grade cases.

It was possible for a woman to have no record of a first or second vaccine dose but records for subsequent doses. In our initial analysis, we assigned exposure status using the actual number of doses that had dates recorded against them, ignoring provider assigned dose numbers. We repeated all analyses after reassigning exposure status based on the last dose number for which there was a valid dose date occurring before the index date, analogous to the "third dose assumption" used to estimate coverage of primary course vaccines in children. ${ }^{17}$

\section{Results \\ Participants}

In total, 108353 women were eligible for inclusion in the primary analysis: $1062(1.0 \%)$ high grade cases, $10887(10.0 \%)$ other cases, and $96404(89.0 \%)$ controls (figure). See supplementary figure 1 on bmj.com for the numbers included in the secondary analysis. The median follow-up time from study start date to index date for women in the primary analysis was 808 days (interquartile range 456-1131 days) for controls, 654 (313-1038) days for other cases, and 766 (381-1087) days for high grade cases. Cases were older, more disadvantaged, and less likely to live in major cities than controls (table $2 \Downarrow$ ). These differences were more pronounced for high grade cases. Supplementary table 1 on bmj.com presents the characteristics for women in the secondary analysis. Table 1 presents the histological and cytological diagnoses for cases (see supplementary table 2 on bmj.com for secondary analysis). For the primary analysis $11.2 \%$ of high grade cases $(n=119), 18.5 \%$ of other cases $(n=2013)$, and $23.8 \%$ of controls $(n=22987)$ were fully vaccinated ( $\geq 3$ doses) before their index date (figure). Fully vaccinated women were younger at first vaccine dose (median age 17.0 years (interquartile range 16.0-19.4 years) than partially vaccinated women $(19.6(18.0-22.4)$ years for two doses; 21.0 (19.1-23.7) years for one dose).

\section{Vaccine effectiveness estimates}

The largest difference between crude and adjusted estimates was made by adjusting for year of birth. Effect estimates were essentially unchanged after adjustment for remoteness and socioeconomic status in both the primary and secondary analysis. 
The primary analysis was of women who had no cytology tests before their index date. In this analysis, the adjusted odds ratios for exposure to three doses of HPV vaccine compared with no vaccine were 0.54 (95\% confidence interval 0.43 to 0.67 ) for high grade cases and 0.66 ( 0.62 to 0.70$)$ for other cases compared with controls with normal cytology (table $3 \Downarrow)$, equating to vaccine effectiveness of $46 \%$ (95\% confidence interval $33 \%$ to $57 \%$ ) and $34 \%$ (30\% to 38\%), respectively. The adjusted numbers needed to vaccinate (three doses) to prevent one cervical abnormality at first screening round were 125 (95\% confidence interval 97 to 174) and 22 (19 to 25), respectively. We observed variation in estimates across age strata, with significantly reduced odds of full vaccination in high grade cases aged 15 to 18 years and 19 to 22 years, and little evidence of any vaccine effect among vaccinated women in the oldest age stratum (exposure odds ratio $0.95,95 \%$ confidence interval 0.63 to 1.45 ). The adjusted odds ratios for exposure to two vaccine doses were 0.79 ( 0.64 to 0.98$)$ for high grade cases and 0.79 ( 0.74 to 0.85$)$ for other cases, equating to vaccine effectiveness of $21 \%$ (table 3 ). We found no statistically significant effectiveness of one dose for high grade or other cases. Adjusted effectiveness of one or more vaccine doses against a high grade cervical abnormality was $26 \%$ (15\% to $36 \%$ ) and against any other abnormality was $22 \%$ (18\% to $25 \%$ ).

The secondary analysis was of women who had one or more cytology tests before their index date. For this analysis the adjusted odds ratios for exposure to three doses of HPV vaccine compared with no vaccine were 0.77 (0.64 to 0.92 ) for high grade cases and 0.81 ( 0.72 to 0.91 ) for other cases (see supplementary table 3 on bmj.com). The adjusted numbers needed to vaccinate were 38 (23 to 104) and 24 (16 to 50), respectively. Effectiveness of two doses did not reach statistical significance. Adjusted effectiveness of one or more vaccine doses against a high grade cervical abnormality was $18 \%$ (4\% to $30 \%)$ and against any other abnormality was $15 \%(6 \%$ to $23 \%)$.

\section{Sensitivity analyses}

Vaccine effectiveness estimates for three doses were essentially unchanged when we assumed latent periods of 30, 180, and 365 days (see supplementary tables 4-6 on bmj.com); however, the effectiveness of two doses seemed to increase with assumed latent periods of 180 and 365 days.

Vaccine effectiveness remained essentially unchanged in sensitivity analyses in which we used last dose number to assign exposure status rather than number of doses recorded on the register (see supplementary table 7 on bmj.com). Finally, in the secondary analysis, vaccine effectiveness was also unchanged when we defined the index date for high grade cases as the date of their first ever abnormal cytology test result in the study period rather than the cytology test result immediately preceding the abnormal histology test result (data not shown).

\section{Discussion}

\section{Key findings and comparison with other studies}

Four years after the introduction of a routine and catch-up vaccination programme against human papillomavirus (HPV), we estimated that the quadrivalent vaccine provided $46 \%$ protection against histologically confirmed high grade cervical abnormalities and $34 \%$ protection against other cervical abnormalities in women who had not started screening before vaccination. The numbers needed to vaccinate to prevent one cervical abnormality at first screening round were 125 for a histologically confirmed high grade abnormality and 22 for other abnormalities. We found that partial vaccination with two doses provided $21 \%$ protection against both high grade and other cervical abnormalities.

These findings are in accord with recent studies indicating that approximately $52 \%$ of high grade cervical abnormalities are due to HPV 16 and HPV $18 .^{18}$ This attributable fraction is the theoretical upper limit of vaccine efficacy, unless cross protection against other HPV types provides additional protection. Vaccine effectiveness in the population setting would therefore be expected to be lower than this ideal, owing to imperfect implementation, previous exposure, and other factors. ${ }^{19}$

Our vaccine effectiveness estimates for one or more vaccine doses (primary analysis: 26\% against high grade abnormality, $22 \%$ against any other abnormality; secondary analysis: $18 \%$ against high grade abnormality, $15 \%$ against any other abnormality) are most comparable, and are similar, to phase III trial intention to treat analyses, which reported efficacy of one or more vaccine doses against all HPV related disease (including disease cases due to non-vaccine type) in the population that included women already infected with vaccine type HPV but without disease at recruitment. In women vaccinated with one or more doses the proportion of histologically confirmed high grade lesions decreased by $19 \%$ (95\% confidence interval $7.7 \%$ to $28.9 \%$ ) and all categories of cervical smear abnormalities decreased by $11.3 \%$ (6.5\% to $15.9 \%) .{ }^{20}$ Both the slightly longer follow-up time and the differences between our population and the trial populations could account for the higher effectiveness estimates in our primary analysis. Our population may contain a higher proportion of HPV naïve women than the trial populations because we excluded women who had attended screening before their index date. Women new to screening may be closer to sexual debut than women who have been regularly screened and for longer, despite having a history of only negative cytology results. Both relative and absolute estimates of vaccine effectiveness are encouraging after a short duration following the introduction of the national programme.

Data on the effectiveness of partial vaccination against cervical abnormalities are limited as such efficacy was not assessed in clinical trials. Although this analysis indicates that three vaccine doses are required for optimal protection, our data suggest reasonable effectiveness with fewer doses. Partially vaccinated women were older than fully vaccinated women and therefore more likely to have been infected with HPV before vaccination. We may therefore have underestimated the effectiveness of partial vaccination. Further research is needed to establish whether optimised two dose schedules are adequate.

We found that relative vaccine effectiveness was lower in the secondary analysis in the population of women with one or more cytology test results before their index date (that is, who met the case definition on subsequent screening rounds) compared with women in the primary analysis with no previous tests. High grade cases in particular were statistically significantly more likely to have had one or more previous tests. Australian guidelines recommend that women who have a low grade abnormality on cervical smear testing should undergo repeat testing to assess clearance (usually at 12 months) before proceeding to colposcopy and biopsy. Because of these screening recommendations, and given the clinical course of HPV infection, it is not surprising that women with high grade abnormalities are more likely than other women to have had preceding cytology tests. We found that the number needed to vaccinate to prevent one high grade cervical abnormality was lower among women with a screening history than among women with no previous screening history ( $38 v 125)$. This is 
likely to be because the incidence of high grade abnormalities is higher in this group of women and reflects the fact that unlike the exposure odds ratio, the number needed to vaccinate is a measure that is influenced by both effect size and outcome incidence. Our finding also accords with a subanalysis from the trials indicating a benefit of vaccination even in women already treated for cervical disease. ${ }^{21}$ The number needed to vaccinate to prevent one other cervical abnormality, however, was similar in women both with and without a history of smear tests $(24 v$ 22).

\section{Strengths and limitations of this study}

Our study used administrative databases, which avoid some of the reporting and selection biases that can occur in interview based studies. Inclusion of both the school and the community based catch-up programmes enabled a comprehensive evaluation of the effect of the vaccination with adequate power to assess outcomes for those women who only received one or two doses. Consistent with known risk factors for cervical abnormalities, cases were older, more disadvantaged, and less likely to live in major cities than controls. Because age, socioeconomic status, and remoteness were associated with case status and are likely to be associated with cervical screening, we stratified our analyses by age group and included terms for year of birth, measures of socioeconomic status and remoteness, and index date. We used cumulative incidence sampling in which controls were defined by having no abnormal cytology test results throughout the study period. We were concerned about possible exposure opportunity bias, whereby control participants may have had systematically greater opportunity for vaccination owing to longer duration in the cohort overall. We controlled for this in two ways. Firstly, we included a term for follow-up time in the analysis. Including this term in the primary analysis attenuated estimates of vaccine effectiveness by up to $20 \%$ for other cases, but had a negligible impact on estimates for high grade cases. Secondly, in our secondary analysis, among control women (defined as having persistently negative smear test results), the index date was randomly selected from among their negative screens using a computer algorithm.

Our study has some limitations. Misclassification of exposure status in some women is likely to have occurred owing to inaccuracies in vaccine register data and those arising from data linkage. One quarter of women aged $19-26$ years and $12 \%$ of women aged 12-18 years recorded on the vaccine register had no record of a first dose but had records of subsequent doses. This suggests either inaccuracy in reporting of dose numbers or under-reporting of vaccine doses by providers to the vaccine register. Proportions of women classed as vaccinated in our study are lower than national coverage statistics because our study included screening events that occurred during the delivery phase of the vaccination programme, and we counted only vaccine doses delivered before a woman's index date. Some degree of under-linkage (failure to correctly assign a woman's vaccination record to her smear test record) may have occurred during probabilistic linkage between registers owing to possible address or name changes as a result of marriage in this particular population. Over-linkage (that is, assigning a vaccination history to a woman erroneously) should, however, occur infrequently. ${ }^{22}$ We believe that under-linkage is likely to have occurred infrequently and to have been non-differential for cases and controls. Non-differential under-linkage or under-reporting could lead to underestimation of vaccine effectiveness. However it is possible that under-reporting occurred differentially because cases were older and therefore more likely to have been vaccinated as part of the community based programme (where under-reporting was higher). The potential impact of this would be to over-estimate vaccine effectiveness. We tested the impact of under-reporting of doses in sensitivity analyses, where possible, and found no statistically significant differences in our results (see supplementary table 7 on bmj.com).

We were able to control for a limited number of covariates in this analysis. It is possible that residual confounding by other factors may have occurred (for example, smoking or sexual activity); however, we consider it unlikely that any such confounding would be of sufficient magnitude and in the direction observed to explain these findings. Finally, generalisability of our findings to other population settings may be limited. Vaccine effectiveness will predominantly depend on the extent of previous infection and type specific HPV prevalence in the population cohort vaccinated. This will relate to age of sexual debut in relation to age at vaccination and vaccine coverage. Indeed, while we conclude that estimates of vaccine effectiveness in our youngest age group (11 to 14 years) are likely to be generalisable to the cohort of 12 year old girls targeted by the ongoing vaccination programme, it might be anticipated that effectiveness in this cohort of girls will be higher.

\section{Conclusions and implications for policy}

These population data suggest a risk reduction of $46 \%$ for histologically confirmed high grade cervical abnormalities and $34 \%$ for other cervical abnormalities among young women who were fully vaccinated with the quadrivalent HPV vaccine before starting screening. The adjusted numbers needed to vaccinate (three doses) to prevent one cervical abnormality at first screening round were 125 and 22 , respectively.

At present, cervical screening will remain necessary in vaccinated populations owing to the relative type specificity of current vaccines. Falling population prevalence of cervical abnormalities will reduce the sensitivity and positive predictive value of cytological testing, ${ }^{23}$ and screening programmes will need to adapt to maintain their effectiveness. Our data suggest that this task is now becoming more urgent given the relatively rapid impact of the vaccine on disease in the real world setting. To tackle this, Australia is currently conducting a comprehensive review of cervical screening. ${ }^{24}$ Continued observation of this population is necessary to assess the implications for cervical screening recommendations in the coming era of mass vaccination.

We thank the following units within Queensland Health for providing the data and conducting the linkage for this study: Communicable Diseases Branch, Cancer Screening Services Branch, Health Statistics Centre. We thank Claire DeBats, Nathan Dunn, Belinda Eagle, and Vicki Bryant for the data extraction. The provision of linked data was made possible through HealthLinQ, the Queensland node of the Population Health Research Network, funded by the Australian Government's National Collaborative Research Infrastructure Strategy. This project was overseen by a steering group and we thank the following members for their contributions: Christine Selvey, Jennifer Muller, and Leane Christie. We thank Paddy Farrington for discussions on study design and analysis and Tim Patterson for his comments on earlier drafts. EC thanks the South East of Scotland Postgraduate Deanery and NHS Borders for support to undertake this research during postgraduate training.

Contributors: EC, AJD, SK, and DCW designed the study. EC is guarantor. EC and NP analysed the data. EC, JMLB, AJD, SK, SBL, $N P$, and DCW interpreted the data. EC wrote the first draft. JMLB, AJD, SK, SBL, NP, and DCW made substantial contributions to drafts of the 


\section{What is already known on this topic}

Vaccination programmes against human papillomavirus (HPV) have been implemented in over 40 countries

Although results from phase III trials show $98 \%$ efficacy of the quadrivalent vaccine against vaccine type related high grade cervical abnormalities, less is known about vaccine effectiveness against cervical abnormalities in population settings

\section{What this study adds}

The quadrivalent HPV vaccine was $46 \%$ effective against high grade cervical abnormalities and $34 \%$ effective against any other cervical abnormality in young women attending their first cervical screening after they were fully vaccinated at school or up to 27 years of age Receipt of two vaccine doses provided some, although lesser, protection

The vaccine seemed more effective for preventing high grade abnormalities than other abnormalities and more effective in younger than older women

manuscript and critically revised the report. All authors saw and approved the final version of the report.

Funding: No specific project funding was received but EC was supported by a salary from the University of Queensland and NHS Borders. DCW was supported by a Future Fellowship from the Australian Research Council (FT0990987). NP was supported by an early career fellowship from the National Health and Medical Research Council (631691). SK and AJD were supported by salaries from the University of Queensland. JMLB was supported by salary from the Victorian Cytology Service. SBL was supported by an early career fellowship from the National Health and Medical Research Council (GNT1036231) and is a Queensland Children's Medical Research Institute senior research fellow supported by the Children's Health Foundation Queensland (50025).

Competing interests: All authors have completed the ICMJE uniform disclosure form at www.icmje.org/coi_disclosure.pdf (available on request from the corresponding author) and declare: no support from any organisation for the submitted work. SBL has received research grants, travel funds, and payments from Merck, GSK, and BioCSL. JMLB has been a co-investigator on Australian HPV epidemiology grants that have received partial unrestricted funding from BioCSL and Merck. The University of Queensland receives royalties from the sale of Gardasil (Merck). There are no other relationships or activities that could appear to have influenced the submitted work.

Ethical approval: This study was approved by the University of Queensland medical research ethics committee (2010001247) and Queensland Health human research ethics committee (HREC/10/QHC/48).

Data sharing: No additional data available.

Transparency: EC affirms that the manuscript is an honest, accurate, and transparent account of the study being reported; that no important aspects of the study have been omitted; and that any discrepancies from the study as planned (and, if relevant, registered) have been explained.

1 Garland SM, Hernandez-Avila M, Wheeler CM, Perez G, Harper DM, Leodolter S, et al. Quadrivalent vaccine against human papillomavirus to prevent anogenital diseases. $N$ Engl J Med 2007;356:1928-43.

2 FUTURE II Study Group. Quadrivalent vaccine against human papillomavirus to prevent high-grade cervical lesions. N Engl J Med 2007;356:1915-27.

3 Ault KA. Effect of prophylactic human papillomavirus $L 1$ virus-like-particle vaccine on risk of cervical intraepithelial neoplasia grade 2 , grade 3 , and adenocarcinoma in situ: a combined analysis of four randomised clinical trials. Lancet 2007;369:1861-8.

4 Paavonen J, Jenkins D, Bosch FX, Naud P, Salmerón J, Wheeler CM, et al. Efficacy of a prophylactic adjuvanted bivalent $L 1$ virus-like-particle vaccine against infection with human papillomavirus types 16 and 18 in young women: an interim analysis of a phase III double-blind, randomised controlled trial. Lancet 2007;369:2161-70.

5 Paavonen J, Naud P, Salmerón J, Wheeler CM, Chow S-N, Apter D, et al. Efficacy of human papillomavirus (HPV)-16/18 AS04-adjuvanted vaccine against cervical infection and precancer caused by oncogenic HPV types (PATRICIA): final analysis of a double-blind, randomised study in young women. Lancet 2009;374:301-14.

6 Wheeler CM, Kjaer SK, Sigurdsson K, Iversen O-E, Hernandez-Avila M, Perez G, et al. The impact of quadrivalent human papillomavirus (HPV; types 6, 11, 16, and 18) L1 virus-like particle vaccine on infection and disease due to oncogenic nonvaccine HPV types in sexually active women aged 16-26 years. $J$ Infect Dis 2009;199:936-44.

7 Bonanni P, Boccalini S, Bechini A. Efficacy, duration of immunity and cross protection after HPV vaccination: a review of the evidence. Vaccine 2009;27(Suppl 1):A46-53.

8 Markowitz LE, Tsu V, Deeks SL, Cubie H, Wang SA, Vicari AS, et al. Human papillomavirus vaccine introduction - the first five years. Vaccine 2012;30(Suppl 5):F139-48.

9 Gertig DM, Brotherton JML, Saville M. Measuring human papillomavirus (HPV) vaccination coverage and the role of the National HPV Vaccination Program Register, Australia. Sex Health 2011;8:171-8.

10 National Cervical Screening Program, Australia. 2013. www.cancerscreening.gov.au/ internet/screening/publishing.nsf/Content/NCSP-Policies-1.

11 Queensland Government. The Queensland Cervical Screening Program and Pap Smear Register 10 year report 1999-2009. www.health.qld.gov.au/cervicalscreening/.

12 Queensland Health. The Queensland Cervical Screening Program statistical report 2004-2006. 2012. www.health.qld.gov.au/cervicalscreening/documents/cerv_stat report0406.pdf.

13 Chief Medical Officer, Department of Health and Ageing, Australian Government. Guidance on revaccination where $\mathrm{HPV}$ vaccine doses have been given at less than recommended minimum intervals. 2009. www.health.gov.au/internet/immunise/publishing.nsf/Content/ cmo-full-advice-hpv-cnt/\$File/CMO-full-advice-hpv.pdf.

14 Bender R, Blettner M. Calculating the "number needed to be exposed" with adjustment for confounding variables in epidemiological studies. J Clin Epidemiol 2002;55:525-30.

15 Woodman CBJ, Collins SI, Young LS. The natural history of cervical HPV infection: unresolved issues. Nat Rev Cancer 2007;7:11-22.

16 Winer RL, Kiviat NB, Hughes JP, Adam DE, Lee S-K, Kuypers JM, et al. Development and duration of human papillomavirus lesions, after initial infection. $J$ Infect Dis 2005;191:731-8.

17 Hull BP, Lawrence GL, MacIntyre CR, McIntyre PB. Estimating immunisation coverage: is the 'third dose assumption' still valid? Commun Dis Intell 2003;27:357-61.

18 Smith JS, Lindsay L, Hoots B, Keys J, Franceschi S, Winer R, et al. Human papillomavirus type distribution in invasive cervical cancer and high-grade cervical lesions: a meta-analysis update. Int J Cancer 2007;121:621-32.

19 Gertig DM, Brotherton JM, Budd AC, Drennan K, Chappell G, Saville AM. Impact of a population-based HPV vaccination program on cervical abnormalities: a data linkage study. BMC Med 2013;11:227.

20 Muñoz N, Kjaer SK, Sigurdsson K, Iversen O-E, Hernandez-Avila M, Wheeler CM, et al. Impact of human papillomavirus (HPV)-6/11/16/18 vaccine on all HPV-associated genital diseases in young women. J Natl Cancer Inst 2010;102:325-39.

21 Joura EA, Garland SM, Paavonen J, Ferris DG, Perez G, Ault KA, et al. Effect of the human papillomavirus (HPV) quadrivalent vaccine in a subgroup of women with cervical and vulvar disease: retrospective pooled analysis of trial data. BMJ 2012;344:e1401.

22 Nitsch D, Morton S, DeStavola BL, Clark H, Leon DA. How good is probabilistic record linkage to reconstruct reproductive histories? Results from the Aberdeen children of the 1950s study. BMC Med Res Methodol 2006;6:15.

23 Castle PE, Schmeler KM. Optimizing cervical cancer prevention strategies in the United States. Gynecol Oncol 2012;127:437-9.

24 Australian Government Department of Health. National Cervical Screening Program Renewal. 2013. www.cancerscreening.gov.au/internet/screening/publishing.nst/Content/ ncsp-renewal.

Accepted: 31 January 2014

\section{Cite this as: BMJ 2014;348:g1458}

This is an Open Access article distributed in accordance with the Creative Commons Attribution Non Commercial (CC BY-NC 3.0) license, which permits others to distribute, remix, adapt, build upon this work non-commercially, and license their derivative works on different terms, provided the original work is properly cited and the use is non-commercial. See: http://creativecommons.org/licenses/by-nc/3.0/. 


\section{Tables}

\section{Table 1| Frequency of cytological and histological diagnoses for cases, 1 April 2007 to 31 March 2011, primary analysis}

\begin{tabular}{|c|c|}
\hline Cervical abnormality* by case group and diagnosis & No (\%) \\
\hline \multicolumn{2}{|l|}{ High grade cases $\uparrow(n=1062)$} \\
\hline Histologically confirmed high grade cervical abnormality: & $1062(100)$ \\
\hline \multicolumn{2}{|l|}{ Squamous abnormalities: } \\
\hline Squamous cell carcinoma, invasiveł & $7(0.7)$ \\
\hline CIN 3 & $944(88.9)$ \\
\hline CIN 2 & $84(7.9)$ \\
\hline CIN not otherwise specified & $15(1.4)$ \\
\hline \multicolumn{2}{|l|}{ Endocervical abnormalities: } \\
\hline Adenocarcinoma, microinvasive & $1(0.1)$ \\
\hline Adenocarcinoma in situ & $11(1.0)$ \\
\hline \multicolumn{2}{|l|}{ Other cases $(n=10887)$} \\
\hline Histologically confirmed low grade abnormality: & $903(8.3)$ \\
\hline \multicolumn{2}{|l|}{ Squamous abnormalities: } \\
\hline Low grade squamous abnormality & $903(8.3)$ \\
\hline Cytological abnormality only: & $9984(91.7)$ \\
\hline \multicolumn{2}{|l|}{ Cytological squamous abnormalities: } \\
\hline HSIL with possible microinvasion or invasion & $1(0.009)$ \\
\hline HSIL & $428(3.9)$ \\
\hline Possible HSIL & $293(2.69)$ \\
\hline LSIL & $4692(43.1)$ \\
\hline Possible LSIL & $4547(41.8)$ \\
\hline \multicolumn{2}{|l|}{ Cytological endocervical abnormalities§: } \\
\hline Adenocarcinoma in situ & $1(0.009)$ \\
\hline Possible high grade endocervical abnormality & $2(0.02)$ \\
\hline Atypical endocervical cells of unknown significance & $20(0.2)$ \\
\hline
\end{tabular}

$\mathrm{CIN}=$ cervical intraepithelial neoplasia; HSIL=high grade squamous intraepithelial lesion; LSIL=low grade squamous intraepithelial lesion.

${ }^{*} H i s t o l o g y$ and cytology reporting categories of the Australian national cervical screening programme. The Australian modified Bethesda System 2004 designates atypical cells of undetermined significance as possible LSIL and atypical squamous cells-cannot exclude HSIL as possible HSIL.

tIndex date for high grade cases is date of cytology test immediately preceding the histology test.

‡Of the seven women with squamous cell carcinoma (invasive), five were unvaccinated, one received one dose, and one received two doses before her index date.

§Five women designated as "other cases" had mixed squamous and endocervical abnormalities and were classified according to the endocervical component: atypical endocervical cells of uncertain significance (two had possible HSIL, two had possible LSIL, and one had LSIL). 
Table 2/ Comparison of characteristics of cases and controls, 1 April 2007 to 31 March 2011, primary analysis. Values are numbers (percentages) unless stated otherwise

\begin{tabular}{|c|c|c|c|}
\hline Categories & Controls ( $n=96404)$ & Other cases ( $n=10887$ ) & High grade cases $(n=1062)$ \\
\hline \multicolumn{4}{|l|}{ Age (years) in $2007^{\star}$ : } \\
\hline $11-14$ & $2525(2.6)$ & $291(2.7)$ & $13(1.2)$ \\
\hline $15-18$ & $32044(33.2)$ & $4100(37.7)$ & $213(20.1)$ \\
\hline $19-22$ & $33568(34.8)$ & $4187(38.5)$ & $423(39.8)$ \\
\hline $23-27$ & $28267(29.3)$ & $2309(21.2)$ & $413(38.9)$ \\
\hline \multicolumn{4}{|l|}{ Socioeconomic fifth: } \\
\hline 1 (most disadvantaged) & $12403(12.9)$ & $1415(13.0)$ & $173(16.3)$ \\
\hline 2 & $17938(18.6)$ & $2078(19.1)$ & $211(19.9)$ \\
\hline 3 & $23021(23.9)$ & $2775(25.5)$ & $268(25.2)$ \\
\hline 4 & $25268(26.2)$ & $2860(26.3)$ & $264(24.9)$ \\
\hline 5 (least disadvantaged) & $17774(18.4)$ & $1759(16.2)$ & $146(13.7)$ \\
\hline \multicolumn{4}{|l|}{ Remoteness category: } \\
\hline Major cities of Australia & $64286(66.7)$ & $6980(64.1)$ & $646(60.8)$ \\
\hline Inner regional Australia & $16025(16.6)$ & $1770(16.3)$ & $180(16.9)$ \\
\hline Outer regional Australia & $14216(14.7)$ & $1807(16.6)$ & $207(19.5)$ \\
\hline Remote/very remote Australia & $1877(1.9)$ & $330(3.0)$ & $29(2.7)$ \\
\hline \multicolumn{4}{|l|}{ Follow-up periodst: } \\
\hline Study start date to index date & $808(456-1131)$ & $654(313-1038)$ & $766(381-1087)$ \\
\hline Study start date to first vaccine dose & $211(60-372)$ & $208(61-365)$ & $248(165-431)$ \\
\hline Study start date to last vaccine dose & $348(211-559)$ & $334(207-537)$ & $393(229-570)$ \\
\hline First vaccine dose to index date & 614 (293-932) & $492(195-861)$ & $444(150-801)$ \\
\hline Last vaccine dose to index date & $442(145-759)$ & $333(93-676)$ & $326(70-633)$ \\
\hline \multicolumn{4}{|l|}{ Follow-up quarter (time to index date): } \\
\hline 1 (0-494 days) & $22991(23.8)$ & $3816(35.1)$ & $312(23.4)$ \\
\hline 2 (495-856 days) & $24175(25.1)$ & $2721(25.0)$ & $241(22.7)$ \\
\hline 3 (856-1165 days) & $24524(25.4)$ & $2221(20.4)$ & $279(26.3)$ \\
\hline 4 (>1165 days) & $24713(25.6)$ & $2129(19.6)$ & $230(21.7)$ \\
\hline
\end{tabular}

*Australian Bureau of Statistics estimated resident populations for Queensland women in 2007 were 111805 (11-14 years), 112250 (15-18 years), 113920 (19-22 years), and 142836 (23-27 years).

†Median (interquartile range) time (days). 


\begin{tabular}{|c|c|c|c|c|c|c|c|}
\hline \multirow{2}{*}{$\begin{array}{l}\text { No of } \\
\text { doses, by } \\
\text { age in } 2007\end{array}$} & \multirow[b]{2}{*}{$\begin{array}{l}\text { No }(\%) \text { of } \\
\text { controls }\end{array}$} & \multicolumn{3}{|c|}{ Other cases } & \multicolumn{3}{|c|}{ High grade cases } \\
\hline & & No (\%) & $\begin{array}{l}\text { Crude odds ratio* } \\
\text { (95\% Cl) }\end{array}$ & $\begin{array}{l}\text { Adjusted odds ratio } \neq \\
(95 \% \mathrm{Cl})\end{array}$ & No (\%) & $\begin{array}{l}\text { Crude odds ratio } † \\
(95 \% \mathrm{Cl})\end{array}$ & $\begin{array}{l}\text { Adjusted odds ratio } \\
(95 \% \mathrm{Cl})\end{array}$ \\
\hline \multicolumn{8}{|l|}{ 11-14 years: } \\
\hline 0 & $619(24.5)$ & $102(35.1)$ & reference & reference & $4(30.8)$ & reference & reference \\
\hline 1 & $171(6.8)$ & $24(8.2)$ & 0.85 (0.53 to 1.37$)$ & $0.84(0.52$ to 1.36$)$ & $3(23.1)$ & 2.72 (0.6 to 12.2$)$ & $2.54(0.54$ to 11.8$)$ \\
\hline 2 & 325 (12.9) & $36(12.4)$ & 0.67 (0.45 to 1.01$)$ & 0.67 (0.45 to 1.02$)$ & 0 & - & - \\
\hline 3 & $1410(55.8)$ & $129(44.3)$ & 0.56 (0.42 to 0.73$)$ & $0.60(0.45$ to 0.80$)$ & $6(46.2)$ & 0.66 (0.19 to 2.34$)$ & 0.71 (0.19 to 2.66$)$ \\
\hline \multicolumn{8}{|l|}{ 15-18 years: } \\
\hline 0 & $9918(31.0)$ & $1666(40.6)$ & reference & reference & $101(47.4)$ & reference & reference \\
\hline 1 & $2564(8.0)$ & $435(10.6)$ & $1.01(0.90$ to 1.13$)$ & $0.98(0.87$ to 1.1$)$ & $22(10.3)$ & 0.84 (0.53 to 1.34$)$ & $0.86(0.54$ to 1.37$)$ \\
\hline 2 & $4195(13.1)$ & $538(13.1)$ & 0.76 (0.48 to 1.09$)$ & $0.80(0.72$ to 0.89$)$ & $31(14.6)$ & 0.73 (0.48 to 1.09$)$ & $0.77(0.51$ to 1.16$)$ \\
\hline 3 & 15367 (48.0) & 1461 (35.6) & 0.57 (0.53 to 0.61$)$ & 0.64 (0.59 to 0.69$)$ & $59(27.7)$ & 0.38 (0.27 to 0.52$)$ & $0.43(0.31$ to 0.62$)$ \\
\hline \multicolumn{8}{|l|}{ 19-22 years: } \\
\hline 0 & $20896(62.2)$ & $2891(69.0)$ & reference & reference & $306(72.3)$ & reference & reference \\
\hline 1 & $4230(12.6)$ & $569(13.6)$ & 0.97 (0.88 to 1.07$)$ & $0.98(0.89$ to 1.08$)$ & $46(10.9)$ & 0.74 (0.54 to 1.01$)$ & 0.75 (0.55 to 1.02$)$ \\
\hline 2 & $4254(12.7)$ & $413(9.9)$ & $0.70(0.63$ to 0.78$)$ & $0.80(0.72$ to 0.90$)$ & $42(10.0)$ & 0.67 (0.49 to 0.93$)$ & $0.68(0.49$ to 0.94$)$ \\
\hline 3 & $4188(12.5)$ & $314(7.5)$ & $0.54(0.48$ to 0.61$)$ & $0.70(0.61$ to 0.79$)$ & $29(6.9)$ & $0.47(0.32$ to 0.69$)$ & $0.47(0.32$ to 0.70$)$ \\
\hline \multicolumn{8}{|l|}{ 23-27 years: } \\
\hline 0 & $21599(76.4)$ & $1862(80.6)$ & reference & reference & $318(77.0)$ & reference & reference \\
\hline 1 & $2570(9.1)$ & $202(8.7)$ & 0.91 (0.82 to 1.57$)$ & $0.86(0.74$ to 1.0$)$ & $43(10.4)$ & 1.14 (0.82 to 1.57$)$ & $1.22(0.89$ to 1.7$)$ \\
\hline 2 & $2076(7.3)$ & $136(5.9)$ & $0.76(0.64$ to 0.91$)$ & $0.8(0.67$ to 0.96$)$ & $27(6.5)$ & 0.88 (0.78 to 1.06$)$ & 0.97 (0.65 to 1.45$)$ \\
\hline 3 & $2022(7.2)$ & $109(4.7)$ & $0.63(0.51$ to 0.76$)$ & 0.72 (0.59 to 0.88$)$ & $25(6.1)$ & 0.84 (0.56 to 1.37$)$ & 0.95 (0.63 to 1.45$)$ \\
\hline \multicolumn{8}{|l|}{ All ages: } \\
\hline 0 & $53032(55.0)$ & $6521(59.9)$ & reference & reference & $729(68.7)$ & reference & reference \\
\hline 1 & $9535(9.9)$ & $1230(11.3)$ & 1.05 (0.98 to 1.12$)$ & $0.95(0.89$ to 1.02$)$ & $114(10.7)$ & $0.87(0.71$ to 1.06$)$ & $0.95(0.77$ to 1.16$)$ \\
\hline 2 & $10850(11.3)$ & $1123(10.3)$ & $0.84(0.79$ to 0.90$)$ & 0.79 (0.74 to 0.85$)$ & $100(9.4)$ & 0.67 (0.54 to 0.83$)$ & 0.79 (0.64 to 0.98$)$ \\
\hline 3 & 22987 (23.8) & $2013(18.5)$ & 0.71 (0.68 to 0.75$)$ & $0.66(0.62$ to 0.70$)$ & $119(11.2)$ & 0.38 (0.31 to 0.46$)$ & $0.54(0.43$ to 0.67$)$ \\
\hline
\end{tabular}




\section{Figure}

Eligible population $(n=128039)$

Queensland resident women who attended for first ever cervical smear test between 1 April 2007 and 31 March 2011 and were vaccine eligible during catch-up programme (born July 1980-July 1997)

Excluded $(n=19686)$ :

First presentation for histology test $(n=400)$

Recorded histology test result not preceded by abnormal cytology test result $(n=428)$

Duplicate VIVAS records $(n=29)$

Inconsistent dose sequences $(n=103)$

Not possible to assign to socioeconomic or remoteness category $(n=101)$

$\geq 1$ cytology tests before index date (included in secondary analysis) $(n=18625)$

Study sample population for primary analysis $(n=108353)$

Individual vaccine doses excluded

Duplicate recorded vaccine doses $(n=20)$

Doses breaching recommended minimum dosing intervals:

No of dose 2 doses deleted (dose 1 to 2 breach) $(n=246)$

No of dose 3 doses deleted (dose 2 to 3 breach) $(n=1569)$

No of dose 3 doses deleted (dose 1 to 3 breach) $(n=117)$

Doses with recorded cold chain breach $(n=7)$

\begin{tabular}{l|l|l|} 
& \\
High grade cases $(n=1062):$ & Other cases $(n=10887):$ & \\
0 doses $(n=729,69 \%)$ & 0 doses $(n=6521,60 \%)$ & Controls $(n=96404):$ \\
1 dose $(n=114,11 \%)$ & 1 dose $(n=1230,11 \%)$ & 1 dose $(n=53032,55 \%)$ \\
2 doses $(n=100,9 \%)$ & 2 doses $(n=1123,10 \%)$ & 2 doses $(n=10850,11 \%)$ \\
3 doses $(n=119,11 \%)$ & 3 doses $(n=2013,19 \%)$ & 3 doses $(n=22987,24 \%)$ \\
\hline
\end{tabular}

Eligibility and exclusions, primary analysis. VIVAS=Queensland Health Vaccination Information Vaccination Administration System 